\title{
Adaptive radiotherapy in locally advanced esophageal cancer with atelectasis: a case report
}

\author{
Katsuyuki Sakanaka, Kota Fujii and Takashi Mizowaki
}

\begin{abstract}
Background: To the best of our knowledge, no study has reported mediastinal shift accompanied with obstructive atelectasis due to bulky primary esophageal tumor components treated with adaptive radiotherapy and concurrent chemotherapy.

Case presentation: Here we report the case of a 65-year-old male patient diagnosed with locally advanced thoracic esophageal squamous cell cancer, clinical T4bN1M0, stage IVA. Bronchoscopy and computed tomography (CT) revealed an almost complete obstruction of the lumen of the left bronchus due to compression by bulky primary esophageal tumor components. On admission, the patient presented with dyspnea and decreased arterial oxygen saturation. Chest radiography and $\mathrm{CT}$ on admission revealed mediastinal shift with left atelectasis, as opposed to findings from the chest radiography performed 26 days before admission. Because of the patient's overall good condition, we recommended definitive chemoradiotherapy instead of palliative bronchial stent placement. After obtaining the patient's consent, chemoradiotherapy was initiated on the following day and it comprised three-dimensional conformal radiotherapy with 60 Gy in 30 fractions with concurrent administration of cisplatin and 5-fluorouracil. During chemoradiotherapy, tumor location was monitored with cone-beam CT and chest radiography. Chemoradiotherapy on day 8 revealed no evidence of the mediastinal shift. CT simulation was reperformed to adjust the radiotherapy fields to account for geometrical changes induced by the absence of the mediastinal shift. Subsequently, the mediastinal shift and bronchial obstruction did not recur during the course of chemoradiotherapy. The patient completed the planned radiotherapy with concurrent and adjuvant chemotherapy, and no non-hematological grade $\geq 3$ adverse events were observed. Complete response was confirmed 7 months after initiating chemoradiotherapy. Currently, no disease recurrence, dysphagia, or respiratory symptoms have been reported at 13 months after initiating chemoradiotherapy.
\end{abstract}

Conclusions: In this study, a bulky primary esophageal tumor caused mediastinal shift due to ipsilateral bronchial obstruction. The close follow-up for monitoring resolution of the mediastinal shift during the course of chemoradiotherapy enabled adequate dose delivery to targets, thus reflecting the geometrical changes induced by the absence of the mediastinal shift. Adaptive radiotherapy technique was crucial for favorable patient outcomes in this challenging clinical situation.

Keywords: Esophageal cancer, Mediastinal shift, Adaptive radiotherapy

\footnotetext{
* Correspondence: sakanaka@kuhp.kyoto-u.ac.jp

Department of Radiation Oncology and Image-Applied Therapy, Graduate

School of Medicine, Kyoto University, 54 Shogoin Kawahara-cho, Sakyo-ku,

Kyoto 606-8507, Japan
}

(C) The Author(s). 2020 Open Access This article is distributed under the terms of the Creative Commons Attribution 4.0 International License (http://creativecommons.org/licenses/by/4.0/), which permits unrestricted use, distribution, and reproduction in any medium, provided you give appropriate credit to the original author(s) and the source, provide a link to the Creative Commons license, and indicate if changes were made. The Creative Commons Public Domain Dedication waiver (http://creativecommons.org/publicdomain/zero/1.0/) applies to the data made available in this article, unless otherwise stated. 


\section{Background}

Adaptive radiotherapy aims at adjusting the treatment plan during the course of radiotherapy to ensure correct target coverage and avoid normal tissue complications [1]. Its clinical usefulness has been reported in patients with lung cancer, atelectasis risk, pleural effusion, and obstructive pneumonitis related to lung cancer. During the course of radiotherapy, atelectasis, pleural effusion, and pneumonitis may improve or aggregate, eventually changing the geometrical location of lung tumors. Such geometrical changes in tumor location reportedly result in inaccurate dose delivery to targets and organs at risk during the course of radiotherapy for lung cancer, consequently affecting the clinical outcomes in patients [2]. Adaptive radiotherapy is necessary to adjust the radiotherapy plan when a geometrical change in tumor location occurs during the course of radiotherapy.

To the best of our knowledge, no existing studies in the literature have reported a mediastinal shift accompanied with ipsilateral obstructive atelectasis due to bulky thoracic esophageal cancer. Therefore, the usefulness of adaptive radiotherapy for treating thoracic esophageal cancer with mediastinal shift remains to be explored. The present study reports a case of locally advanced thoracic esophageal cancer with mediastinal shift accompanied with ipsilateral obstructive atelectasis due to primary esophageal tumor components successfully treated with adaptive radiotherapy plus concurrent chemotherapy.

\section{Case presentation}

A 65-year-old male patient with a 3-month history of dysphagia was diagnosed with locally advanced thoracic esophageal squamous cell cancer, cT4bN1M0, stage IVA (Union for International Cancer Control TNM 8th edition) (Fig. 1a and b). The primary esophageal tumor components, including primary esophageal cancer and nearby metastatic lymph nodes, were bulky. The lumen of the left bronchus was almost completely obstructed by compression of the tumor masses (Fig. 1c and d). The patient was referred to our specialized hospital for treating the thoracic esophageal cancer. On the day of admission, he presented with dyspnea and decreased arterial oxygen saturation (approximately 90\% under room air conditions). As opposed to the findings of chest radiography performed 26 days before admission (Fig. 2a), a mediastinal shift with left atelectasis was detected on chest radiography performed on the day of admission (Fig. 2b). Contrast-enhanced computed tomography (CT) revealed that the mediastinal shift was due to complete obstruction of the left bronchus by the primary esophageal tumor components. The patient had a good overall performance status and good organ function immediately before the left bronchial obstruction;

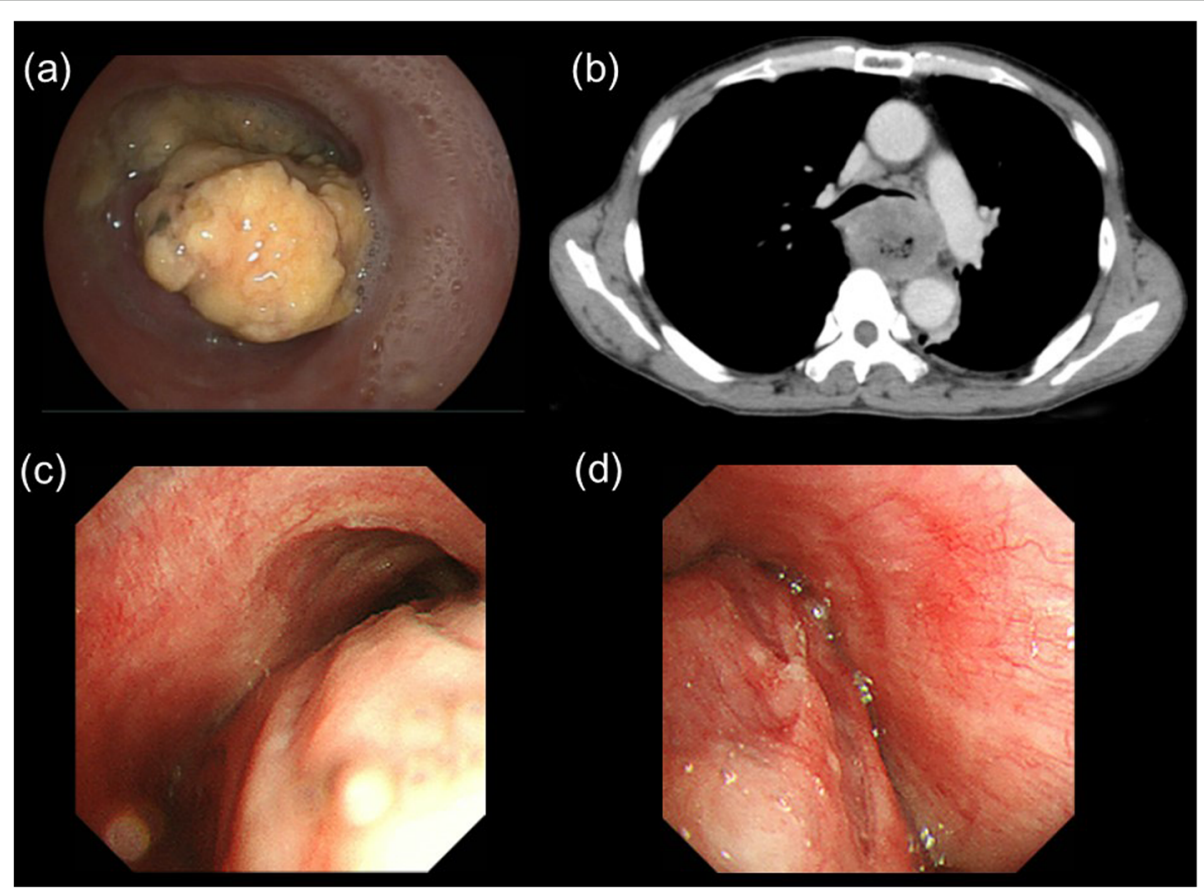

Fig. 1 a Esophagogastroduodenoscopy revealed that the esophageal lumen was filled with necrotic components from the primary esophageal cancer; $\mathbf{b}$ computed tomography $(\mathrm{CT})$ revealed that the left bronchus was compressed by the primary esophageal tumor components; $\mathbf{c}$ bronchoscopy revealed that the tracheal lumen at the carina level was deformed by the primary esophageal tumor components; $\mathbf{d}$ bronchoscopy revealed that the left bronchus presented with an almost complete obstruction due to compression by the primary esophageal tumor components 


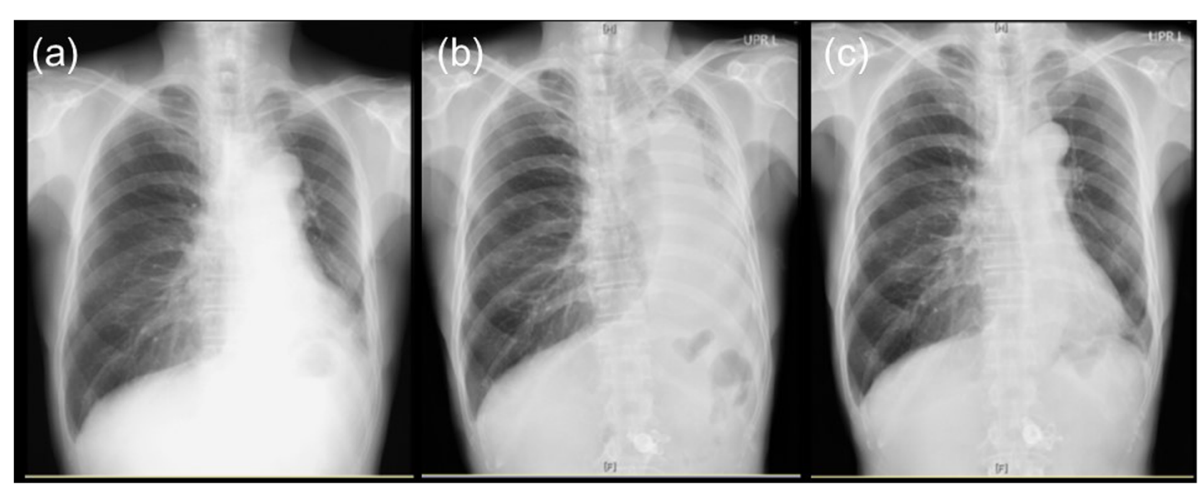

Fig. 2 a Chest radiography performed 26 days before admission revealed no mediastinal shift; $\mathbf{b}$ chest radiography on the day of admission revealed a mediastinal shift and left atelectasis; c chest radiography performed 8 days after initiating chemoradiotherapy revealed no mediastinal shift

therefore, he was recommended to undergo definitive chemoradiotherapy with curative intent rather than palliative bronchial stent placement, and the patient consented to this treatment. Chemoradiotherapy was initiated on the following day and it comprised threedimensional conformal radiotherapy with $60 \mathrm{~Gy}$ in 30 fractions with concurrent administration of cisplatin (70 $\mathrm{mg} / \mathrm{m}^{2}$ on day 1 and 29$)$ and 5 -fluorouracil $\left(700 \mathrm{mg} / \mathrm{m}^{2}\right.$ on days 1-4 and 29-32). Radiotherapy comprised four coplanar irradiation fields, including the gross tumor volumes with adequate margin and elective nodal irradiation for paraesophageal and paratracheal lymph nodes (Fig. 3a-c). Tumor location was monitored with conebeam $\mathrm{CT}$ and chest radiography during the course of chemoradiotherapy. On chemoradiotherapy day 8 , follow-up chest radiography revealed no evidence of the mediastinal shift (Fig. 2c). CT simulation was reperformed for adaptive radiotherapy accounting for the geometrical changes resulting from the absence of the mediastinal shift during the course of chemoradiotherapy. In this manner, the adaptive radiotherapy plan ensured correct dose delivery to the primary esophageal tumor components (Fig. 3d-f). Adaptive radiotherapy improved the target coverage, such that the doses covering $98 \%$ of the planed target volume (D98\%), D50\%, and D2\% were 92, 100, and $105 \%$, respectively, of the prescribed dose in the adaptive radiotherapy plan compared with 7,97 , and $106 \%$, respectively, of the prescribed dose in the nonadaptive radiotherapy plan. After a dose delivery of 40 Gy, the irradiation fields were additionally cone downed to irradiate the gross tumors alone. After completion of the radiotherapy and concurrent chemotherapy plan, two courses of adjuvant chemotherapy were administered every 4 weeks: cisplatin $(80$ $\mathrm{mg} / \mathrm{m}^{2}$ on day 1) and 5 -fluorouracil $\left(800 \mathrm{mg} / \mathrm{m}^{2}\right.$ on days $1-5)$. The patient completed the planned treatment course with chemoradiotherapy and adjuvant chemotherapy with no grade $\geq 3$ non-hematological adverse events. Complete response was confirmed by esophagogastroduodenoscopy and CT performed at 7 months after initiating chemoradiotherapy (Fig. 4a and b). At present, the patient has shown no disease recurrence, dysphagia, or respiratory symptoms at 13 months after initiating chemoradiotherapy. No radiotherapy-related adverse events were observed, except for radiation-induced grade 2 hypothyroidism.

\section{Discussion and conclusions}

The present case report highlights two important clinical observations of our clinical case. Firstly, bronchial obstruction due to a bulky primary esophageal tumor caused a mediastinal shift accompanied with atelectasis. Secondly, adaptive radiotherapy was required to deliver correct doses to the targets, thus reflecting the geometrical changes in tumor location and organs at risk during the course of chemoradiotherapy in patients with thoracic esophageal cancer.

In the present study, bulky primary esophageal cancer components caused a mediastinal shift with bronchial obstruction and atelectasis. Bronchial obstruction, atelectasis, and pleural effusion are well-known associated findings in centrally located lung cancer [2], which have been reported in $23 \%$ of patients with locally advanced lung cancer before initiating radiotherapy by a retrospective study [2]. Contrary to the findings of lung cancer, such findings are rare in untreated esophageal cancer without distant metastases [3]. The major initial symptoms of esophageal cancer are esophagus related, such as dysphagia and weight loss [4], which help in the early diagnosis of esophageal cancer before the primary esophageal tumor components become bulky and compress the bronchi. To the best of our knowledge, the present study is the first report of this nature and it should raise awareness about the fact that left bronchial obstruction may occur due to compression by bulky esophageal primary tumors, causing a mediastinal shift 


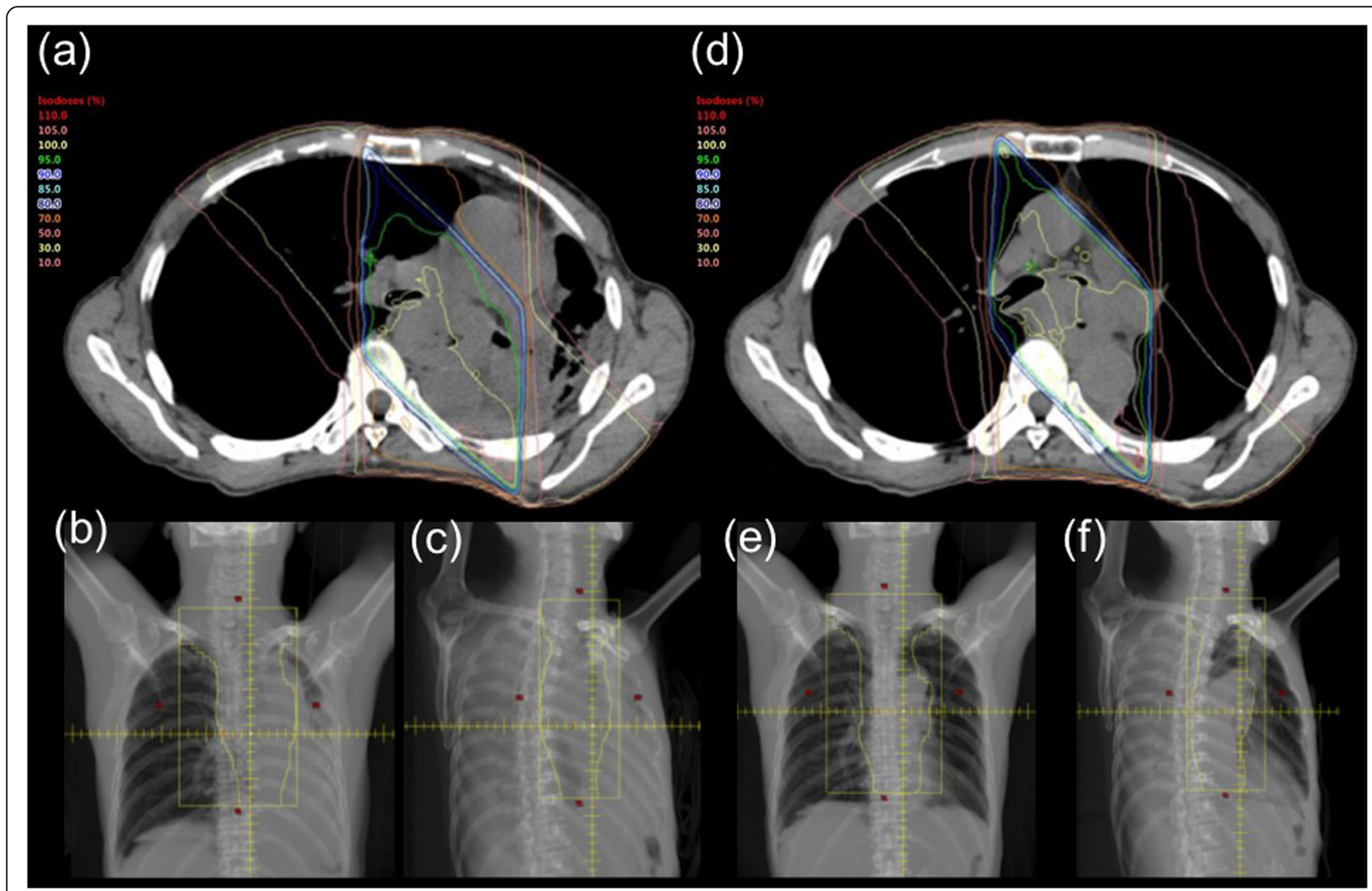

Fig. 3 a Initial radiotherapy plan: axial image of dose distribution (an isodose line of 100\% indicates 2 Gy per fraction); $\mathbf{b}$ initial radiotherapy plan: irradiation field of gantry angle $0^{\circ}$; $\mathbf{c}$ initial radiotherapy plan: irradiation field of gantry angle $315^{\circ}$; $\mathbf{d}$ adaptive radiotherapy plan: axial image of dose distribution showing target coverage as ensured by adaptive radiotherapy reflecting the geometrical change (iso-dose line of 100\% indicates $2 \mathrm{~Gy}$ per fraction); e adaptive radiotherapy plan: irradiation field of gantry angle $0^{\circ} ; \mathbf{f}$ adaptive radiotherapy plan: irradiation field of gantry angle $315^{\circ}$

accompanied with atelectasis in patients with thoracic esophageal cancer.

Chemoradiotherapy for clinical T4 esophageal cancer provides long survival $[5,6]$. Successful tumor shrinkage by chemoradiotherapy for lung cancer reportedly resolves the mediastinal shift and atelectasis by reducing tumor-associated airway obstruction with a geometrical change in the tumor location [7]. The resolution rate of atelectasis after thoracic radiotherapy has been reported to be $38-90 \%$ in patients with lung cancer $[8-10]$. The resolution of atelectasis decreases mass shadow and density in the lung tissue with substantial changes in the normal tissue and tumor doses [11]. Similar to that observed in lung cancer with a mediastinal shift treated

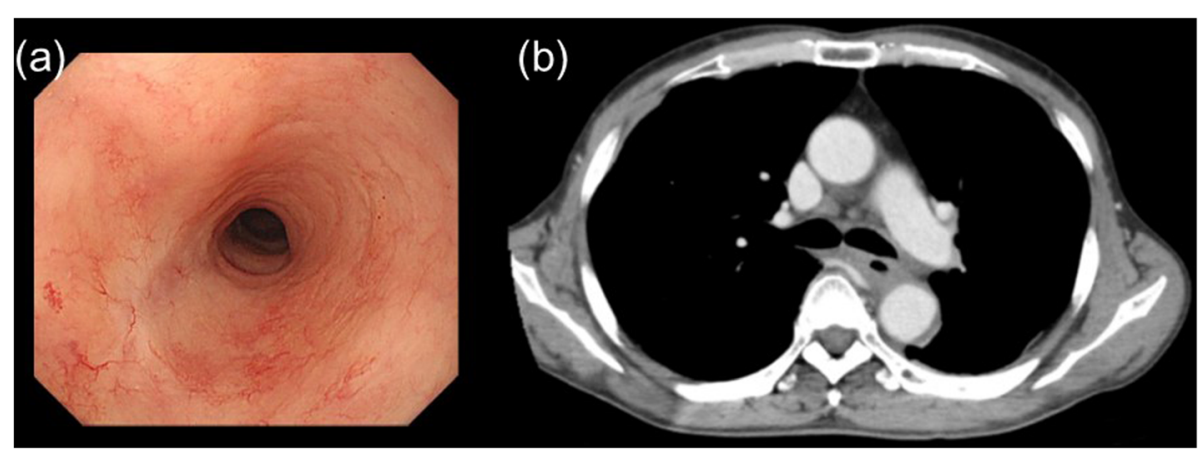

Fig. 4 a Esophagogastroduodenoscopy revealed necrotic components and the disappearance of the esophageal tumor without erosion or ulceration of the esophageal mucosa at 13 months after initiating chemoradiotherapy; $\mathbf{b}$ computed tomography revealed that the airway deformation had resolved and the esophageal tumor was not observed at 13 months after initiating chemoradiotherapy 
with radiotherapy, a geometrical change in the tumor location was observed during the course of chemoradiotherapy in the present study, thus indicating the possibility of inadequate tumor dose coverage. Inadequate dose administration reportedly affects the clinical outcomes of chemoradiotherapy for esophageal cancer [12]. In the clinical case reported in the present study, tumor location was monitored with cone-beam CT and chest radiography during the course of chemoradiotherapy, which enabled the detection of significant geometrical errors in the tumor location outside of the irradiation fields and subsequently lead to correcting these errors through an adaptive radiotherapy plan to deliver an adequate dose to the targets. The present case study demonstrated that chemoradiotherapy with a curative intent is effective for treating locally advanced unresectable esophageal cancer, but this study is unique because it also shows that adaptive radiotherapy warrants an accurate dose delivery to targets in such cases.

In conclusion, a bulky primary esophageal tumor caused mediastinal shift with ipsilateral bronchial obstruction in the present clinical case. A close followup with cone-beam CT or chest radiography during the course of chemoradiotherapy to confirm resolution of the mediastinal shift was key in ensuring the correct dose delivery to targets. Overall, chemoradiotherapy with adaptive radiotherapy appears to yield a curative treatment strategy for patients in such difficult clinical situations.

\section{Abbreviation}

CT: Computed tomography

\section{Acknowledgements}

The authors would like to thank Enago (http://www.enago.jp) for the English language review.

\section{Authors' contributions}

KS participated in the study design, contributed to the radiotherapy planning, and drafted the manuscript. KF contributed to radiotherapy planning. KF and TM drafted the work or substantively revised the manuscript. All the authors have read and approved the final manuscript.

\section{Funding}

English proofreading for the manuscript was supported by Grants-in-Aid for Scientific Research from the Ministry of Education, Culture, Sports, Science, and Technology of Japan under Grant number (17 K16434). The funding body did not participate in the design of the study; collection, analysis, and interpretation of data; or writing the manuscript.

\section{Availability of data and materials}

The datasets used during the current study are available on request from the corresponding author.

\section{Ethics approval and consent to participate}

This study was conducted in accordance with the Declaration of Helsinki and Ethical Guidelines for Medical and Health Research Involving Human Subject in Japan. General written consent for the use of clinical data for research purposes was obtained from the patient before starting radiotherapy and submission of this paper.

\section{Consent for publication}

The patient provided written consent to the use of clinical data for publication.

\section{Competing interests}

The authors declare that they have no competing interests.

Received: 12 April 2019 Accepted: 30 December 2019

Published online: 06 January 2020

References

1. Yan D, Vicini F, Wong J, Martinez A. Adaptive radiation therapy. Phys Med Biol. 1997;42:123-32.

2. Moller DS, Khalil AA, Knap MM, Hoffmann L. Adaptive radiotherapy of lung cancer patients with pleural effusion or atelectasis. Radiother Oncol. 2014; 110:517-22.

3. Herth F KT, Finlay G. Clinical presentation, diagnostic evaluation, and management of central airway obstruction in adults. UpToDate. Waltham, M; UpToDate Inc. https://www.uptodate.com. Accessed 10 Mar 2019.

4. Daly JM, Fry WA, Little AG, Winchester DP, McKee RF, Stewart AK, Fremgen AM. Esophageal cancer: results of an American College of Surgeons patient care evaluation study. J Am Coll Surg. 2000;190:562-72.

5. Sakanaka K, Ishida Y, Itasaka S, Ezoe Y, Aoyama I, Miyamoto S, Horimatsu T, Muto M, Hiraoka M. Identification of a predictive factor for distant metastasis in esophageal squamous cell carcinoma after definitive chemoradiotherapy. Int J Clin Oncol. 2016;21:899-908.

6. Sakanaka K, Ishida Y, Fujii K, Itasaka S, Miyamoto S, Horimatsu T, Muto M, Mizowaki T. Long-term outcome of definitive radiotherapy for cervical esophageal squamous cell carcinoma. Radiat Oncol. 2018;13:7.

7. Tennyson N, Weiss E, Sleeman W, Rosu M, Jan N, Hugo GD. Effect of variations in atelectasis on tumor displacement during radiation therapy for locally advanced lung cancer. Adv Radiat Oncol. 2017;2:19-26.

8. Majid OA, Lee S, Khushalani S, Seydel HG. The response of atelectasis from lung cancer to radiation therapy. Int J Radiat Oncol Biol Phys. 1986;12:231-2.

9. Reddy SP, Marks JE. Total atelectasis of the lung secondary to malignant airway obstruction. Response to radiation therapy. Am J Clin Oncol. 1990;13: 394-400.

10. Vaaler AK, Forrester JM, Lesar M, Edison M, Venzon D, Johnson BE. Obstructive atelectasis in patients with small cell lung cancer. Incidence and response to treatment. Chest. 1997;111:115-20.

11. Guy CL, Weiss E, Jan N, Reshko LB, Christensen GE, Hugo GD. Effect of atelectasis changes on tissue mass and dose during lung radiotherapy. Med Phys. 2016;43:6109.

12. Button MR, Morgan CA, Croydon ES, Roberts SA, Crosby TD. Study to determine adequate margins in radiotherapy planning for esophageal carcinoma by detailing patterns of recurrence after definitive chemoradiotherapy. Int J Radiat Oncol Biol Phys. 2009;73:818-23.

\section{Publisher's Note}

Springer Nature remains neutral with regard to jurisdictional claims in published maps and institutional affiliations.
Ready to submit your research? Choose BMC and benefit from:
- fast, convenient online submission
- thorough peer review by experienced researchers in your field
- rapid publication on acceptance
- support for research data, including large and complex data types
- gold Open Access which fosters wider collaboration and increased citations
- maximum visibility for your research: over $100 \mathrm{M}$ website views per year
At BMC, research is always in progress.
Learn more biomedcentral.com/submissions 